\title{
Transdermal Delivery Enhancement of Gel Containing Niosomes Loaded with Volvariella Volvacea Extract
}

\author{
Warintorn Ruksiriwanich ${ }^{*}$ and Pensak Jantrawut
}

Faculty of Pharmacy, Chiang Mai University, Chiang Mai 50200, Thailand

\begin{abstract}
Our previous study demonstrated the potent in vitro collagen biosynthesis stimulating and antioxidant activities of Volvariella volvacea extract. This study was performed the loading of $V$. volvacea extract (VV) in niosomes (NV). The physicochemical characterization of the VV loaded niosomes and gel containing niosome of the extract (GNV) was studied. Rat skin transdermal absorption by Franz diffusion cells at 6 hours of NV, GNV was compared with $V$. Volvacea extract solution (SV). GNV and NV showed higher chemical stability of total phenolic contents than SV. NV exhibited negative zeta potential values with the mean size of $254 \pm 20.32 \mathrm{~nm}$. Both GNV and NV retarded the cumulative amounts and fluxes of the total phenolic in the extract in the first hour of skin permeation, while enhanced the skin permeation at the $6^{\text {th }}$ hour in the experiment. GNV gave the highest percentages of the total phenolic content through rat skin to the receiving solution followed by $\mathrm{NV}$ and $\mathrm{SV}$, respectively. This study has demonstrated the potential of gel containing niosomes of the $V$. volvacea extract appeared to be the suitable system for topical anti-aging application of $V$. volvacea extract because of the enhancement of chemical stability and rat skin transdermal absorption of $V$. volvacea extract.
\end{abstract}

Keywords: Niosomes, stability, transdermal absorption, Volvariella volvacea.

\section{INTRODUCTION}

Our previous study has demonstrated the potent in vitro collagen biosynthesis stimulating and antioxidant activities of the sonicated aqueous extract of Volvariella volvacea [1] which has known as a paddy straw mushroom or straw mushroom or "Hed Fang" in Thai. It is specie of edible mushroom cultivated throughout the East and Southeast Asia and used extensively in Thai cuisines. V. volvacea (Family: Phuteaceae) is a popular variety among people because of its distinct flavor, pleasant tastes, higher protein content and shorter cropping duration compared to other cultivated mushrooms. Presently, this mushroom is available in most of the supermarkets. It originally grows in rice straw stack in tropical zone that has a high temperature and a rainy climate especially in Chiang Mai, Thailand. Many studies have found that some species of mushrooms are having therapeutic properties such as antioxidant, antimicrobial, anticancer, cholesterol lowering and immuno stimulatory effects [2]. Mushrooms are widely consumed and have been valued as an edible and medical resource. They accumulate a variety of secondary metabolites, including phenolic compounds, polyketides, terpenes and steroids [3]. Mushrooms are traditional in Thailand and also commonly used as food ingredients. Since the potent in vitro collagen biosynthesis stimulating and antioxidant activities of the sonicated aqueous extract of $V$. volvacea $(\mathrm{VV})$, it was loaded in niosomes to enhance its stability, then developed as a cosmetic product and investigated their transdermal delivery [1]. Niosomes are widely used to deliver drugs, cosmetics,

*Address correspondence to this author at the Faculty of Pharmacy, Chiang Mai University, Chiang Mai 50200, Thailand; Tel + 66-86-6076166,

Fax: +66-53-222741; E-mail: warintorn.ruksiri@hotmail.com and plant extracts for pharmaceutical and cosmeceutical purposes [4], especially for the continuous release and targeted delivery [5]. Nonionic surfactants e.g. Span, Tween 61 can be used to form bilayer vesicles because of not only low cost, but also high chemical stability [6]. The bilayer spheroidal structures of these vesicles are usually composed of non-ionic surfactants mixed with cholesterol. These vesicles are advantageous for drug and cosmetic delivery because of their modification of pharmacokinetics, bioavailability and environment protection of the entrapped substances [7, 8]. Transdermal delivery is a convenient method of active ingredient administration that provide the controlled delivery of substance through skin with minimal discomfort [9]. This method offers many benefits over delivery via oral $[10,11]$, intravenous, or injection routes [12, 13] which often result in adverse side effects or fail to deliver the active ingredient to the skin or insufficient concentrations to treat a variety of systemic diseases [14]. However, niosomes were introduced to overcome those problems because niosomes can delivery active ingredient to the target such as anti-cancer drug and anti-infective agent [15].

Thus, the present study has investigated the physicochemical properties and transdermal absorption of the sonicated aqueous extract of $V$. volvacea (VV) loaded niosomes (NV) and gel containing niosomes of VV (GNV) in comparing to VV solution (SV).

\section{MATERIALS AND METHODS}

\section{Materials}

Tween61 (polyoxyethylene sorbitan monostearate), gallic acid, Carbopol® 980, Folin-Ciocalteu reagent and sodium 
carbonate were purchased from Sigma Chemical Co. (St. Louis, MO, USA). Cholesterol from Wako Pure Chemical Industrial Ltd. (Osaka, Japan) was used. All other chemicals and reagents were analytical grade.

\section{Methods}

\section{Preparation of the Sonicated Aqueous Extract of $V$. volvacea $(V V)$}

V. volvacea were collected from Chiang Mai Province in Thailand, during January to June in 2013. The specimen was authenticated by a botanist at Faculty of Pharmacy, Chiang Mai University, Thailand and deposited at Faculty of Pharmacy, Chiang Mai University in Thailand. Then, the mushrooms were washed, cut into pieces, dried at $40 \pm 2{ }^{\circ} \mathrm{C}$ in a hot air oven, ground to powder and kept in an airtight plastic bag at $4 \pm 2{ }^{\circ} \mathrm{C}$ until use. For the extraction process, $100 \mathrm{~g}$ of the dried mushroom powder were sonicated in 1000 $\mathrm{ml}$ of distilled water for $2 \mathrm{~h}$ (with the pulse on for $5.0 \mathrm{sec}$ and off for $2.0 \mathrm{sec})$ at room temperature $\left(25 \pm 2{ }^{\circ} \mathrm{C}\right)$ by the Probe sonicator (Vibra Cell ${ }^{\mathrm{TM}}$, Sonics \& Materials Inc., U.S.A.). The mixtures were filtered through Whatman No. 1 filter paper and the mushroom residues were re-extracted twice under the same conditions. The filtrates were pooled and concentrated under vacuum by a rotary evaporator (R124 Buchi, Switzerland) with the bath temperature of $45 \pm$ $1{ }^{\circ} \mathrm{C}$ and the pressure of $70 \mathrm{mbar}$, and lyophilized. The dried extracts were stored at $4 \pm 2^{\circ} \mathrm{C}$ prior to use. The sonicated aqueous extract of $V$. volvacea $(\mathrm{VV})$ extract was obtained and the percentage yields were calculated on a dry weight basis.

\section{Preparation of Loaded Niosomes}

For VV loaded niosomes (NV), the amounts of $20 \mathrm{mM}$ of Tween61 mixed with cholesterol (at 1:1 molar ratio) were dissolved with chloroform in a round bottom flask. The solvent was removed by a rotary evaporator (Eyela, Tokyo Rikakikai Co. Ltd., Tokyo, Japan) to get a thin film and the residual solvent was dried overnight in a vacuum desiccator. An amount of $20 \mathrm{ml}$ of distilled water together with the VV at various concentrations $(0.5,1.0,2.0$ and $3.0 \% \mathrm{w} / \mathrm{v})$ were added to the film and mixed at $50 \pm 1{ }^{\circ} \mathrm{C}$ for 15 minutes by a rotary evaporator. The dispersion put in the ice bath was sonicated for 2 minutes by an ultrasonic generator (US300T, Nissei, Tokyo, Japan).

\section{Physicochemical Characteristics of the Loaded Niosomes}

The VV loaded niosomes (NV) were stored in transparent vials covered with aluminum cap for 3 months at various temperatures $\left(4 \pm 2,25 \pm 2\right.$ and $\left.45 \pm 2^{\circ} \mathrm{C}\right)$ and investigate at initial and after stored for 1,2 and 3 months. In the evaluating process, the samples were withdrawn and assayed for physical characteristics (appearance, particle size and zeta potential) and the total phenolic contents. Moreover, the NV was evaluated their stability by the accelerated heat cool cycle for 6 cycles.

\section{Physical Characteristics of Niosomes}

\section{The Maximum Loading of the VV}

The concentrations of the $\mathrm{VV}$ entrapped in niosomes were increased from 0.5 to $3.0 \% \mathrm{w} / \mathrm{v}$. The maximum loading of the $\mathrm{VV}$ in niosomes was determined from the maximum concentration of the VV which gave no precipitation.

\section{Appearances}

The NV was investigated for the sedimentation, separation layer and color optically at initial and 1, 2, 3 months.

\section{Particle Sizes}

The particle sizes of various concentrations of VV in NV were measured by dynamic light scattering (DLS) by the Zetasizer 300HSA (Malvern Instruments, Malvern, UK) based on photon correlation spectroscopy. The niosomal dispersions were diluted to 15 times with distilled water. The diameters of loaded niosomes were carried out for $100 \mathrm{~s}$ at room temperature $\left(25 \pm 2^{\circ} \mathrm{C}\right)$. The time-dependent correlation function on the scattered light intensity was measured at a scattering angle of $90^{\circ}$ and wavelength at $535 \mathrm{~nm}$.

\section{Zeta Potential}

The zeta potential values of VV loaded niosomes were determined using the Zetasizer 300HSA (Malvern Instruments, Malvern, UK). The analysis time was kept for $60 \mathrm{~s}$. The average zeta potential was determined. The timedependent correlation function on the scattered light intensity was measured at a scattering angle of $90^{\circ}$. Samples were diluted 15 times with freshly filtrated Millipore water for the particle size and zeta potential measurement.

\section{Preparation of Gel Containing the Niosomes Loaded with VV (GNV)}

The $2.0 \% \mathrm{w} / \mathrm{v}$ of $\mathrm{NV}$ was incorporated into the gel base containing Carbopol® ${ }^{\circledR} 980$. Briefly, 0.3\% (w/w) Carbopol® 980 was dispersed in the niosomal dispersion with gentle stirring, resulting in the gel containing niosomes loaded with $2.0 \%(\mathrm{w} / \mathrm{v})$ of NV (gel of VV niosomes, GNV).

\section{Chemical Stability of the Total Phenolic Acid in Niosomes Loaded with VV (NV) and Gel Containing the Niosomes Loaded with VV (GNV)}

The remaining total phenolic acid contents in the loaded niosomes (NV) and gel containing the niosomes loaded with VV (GNV) were determined at $0,1,2$ and 3 months in comparing to the VV solution (SV) which dissolved in distilled water. Total phenolic contents (TPC) in the form of gallic acid in the GNV, NV and SV were determined using the Folin-Ciocalteu reagent [16]. Briefly, 500 ul of GNV, $\mathrm{NV}$ or SV was extracted by 500 ul of $98 \%$ ethanol, then mixed with $1 \mathrm{~N}$ Folin-Ciocalteu reagent and $20 \% \mathrm{w} / \mathrm{v}$ of 
sodium carbonate $\left(\mathrm{Na}_{2} \mathrm{CO}_{3}\right)$ at ambient temperature $\left(25 \pm 2^{\circ} \mathrm{C}\right)$. After incubation for 30 minutes, the absorbance of blue color developed in each assay mixture was recorded at $760 \mathrm{~nm}$ by a Microplate reader (Model 550, BIORAD, U.S.A.). The TPC in GNV, $\mathrm{NV}$ or $\mathrm{SV}$ were expressed in $\mathrm{mg}$ of gallic acid equivalents (GAE) per gram of extract.

\section{Transdermal Absorption by Vertical Franz Diffusion Cells}

\section{Preparation of the Rat Skin}

The male Sprague-Dawley rats (10-12 weeks, 150-200 mg) were obtained from National Laboratory Animal Centre, Mahidol University, Nakhon Pathom in Thailand. The hair on the abdominal skin was shaved off and left overnight. The rats were sacrificed and the abdominal skin was separated. The subcutaneous fat was then trimmed off by a scalpel. The skin was freshly used. The investigational protocol for all procedures has adhered to the "Principles of Laboratory Animal Care."

\section{Sample Preparation}

The samples used for the transdermal study were gel containing the niosomes loaded with VV (GNV), VV loaded niosomes (NV) and VV solution (SV). All formulations contained $2.0 \% \mathrm{w} / \mathrm{V}$ of $\mathrm{VV}$.

\section{Transdermal Absorption Experiment}

The rat skin was mounted on the receiving compartment solution of the Franz diffusion cells with the stratum corneum (SC) side facing upwards to the donor compartment. One milliliter of each sample was placed in the donor compartment and covered with paraffin film. The available diffusion area of the rat skin was $2.46 \mathrm{~cm}^{2}$. The receiver chamber was filled with $14 \mathrm{ml}$ of phosphate buffer saline ( $\mathrm{pH} 7.4)$, controlled at $37 \pm 2{ }^{\circ} \mathrm{C}$ and constantly stirred at $100 \mathrm{rpm}$ with a small magnetic bar throughout the experiment. The diffusion cells were withdrawn at 1, 3 and 6 hours. Total phenolic contents (TPC) in the whole skin and the receiving compartment solution were extracted and determined using the Folin-Ciocalteu reagent [16]. All experiments were done in triplicate.

\section{Extraction and Determination of TPC in the Treated Samples}

After the experiment, the rat skin was removed from the diffusion cells and swung twice in $100 \mathrm{ml}$ of DI water. The rinsed water was discarded. The skin was cut into small pieces and pooled in a vial containing $5 \mathrm{ml}$ of distilled water. The vial was vortexed for $5 \mathrm{~min}$ and centrifuged at 18,000 rpm, $4{ }^{\circ} \mathrm{C}$ for 10 minutes. The supernatant was collected and dehydrated using a freeze dryer (CHRIST, Martin Christ, Germany). Then, the freezed-dried powder was reconstituted with $1 \mathrm{ml}$ of distilled water and determined for total phenolic contents (TPC) in the form of gallic acid in each sample using the Folin-Ciocalteu reagent.

\section{Data Calculation and Statistical Analysis}

The fluxes at the steady state $\left(\mathrm{mg} / \mathrm{cm}^{2} / \mathrm{h}\right)$, of the samples were calculated using the linear part of the correlation between the cumulative amounts of total phenolic compound $\left(\mathrm{mg} / \mathrm{cm}^{2}\right.$ that permeated the rat skin by unit area and time with the linearity and LLOQ of the cumulative amounts of total phenolic were 0.991 and $0.010 \mathrm{mcg} / \mathrm{ml}$, respectively. Data were expressed as the mean of the three experiments \pm the standard deviation (SD) and were analyzed using ANOVA with LSD test. Statistical analysis differences yielding $p<0.05$ were considered significant.

\section{RESULTS AND DISCUSSION}

\section{Physical Characteristic of Niosomes Loaded with VV Extract (NV)}

Since the sonicated aqueous extracts of $V$. volvacea not only showed the highest total phenolic and polysaccharide contents but also gave the highest DPPH (2,2-diphenyl-1picrylhydrazyl) radical scavenging, lipid peroxidation inhibition and collagen biosynthesis stimulating activities in our previous study [1], VV extract was selected to develop as a topical anti-aging cosmetic product in this study. The nanotechnology, niosomes, was introduced not only to enhance the stability of loaded substance but also modify its pharmacokinetics and bioavailability [8]. Moreover, the niosomes can protect VV extract from the environment, light, heat, and others chemicals that leads to the oxidation process.

\section{The Maximum Loading of VV in Niosomes}

VV extract was loaded in neutral niosomes $(20 \mathrm{mM}$ of Tween61 mixed with cholesterol at 1:1 molar ratio) which was the best formulation from our previous study [17]. When the loading concentration of $\mathrm{VV}$ was more than $2.0 \%(\mathrm{w} / \mathrm{v})$ of $\mathrm{VV}$, the sedimentation of $\mathrm{VV}$ extract was observed at the bottom of bottle. Thus, the maximum loading of $\mathrm{VV}$ in niosomes was $2.0 \%(\mathrm{w} / \mathrm{v})$. VV extract may localize at the hydrophilic core of niosomes and slowly release to the environment. Niosomes loaded with VV extract at $2.0 \% \mathrm{w} / \mathrm{v}$ (NV) was a light brown opalescent suspension

\section{Particle Sizes and Zeta Potential of Niosomes Loaded with $V \boldsymbol{V} \operatorname{Extract}(\mathrm{NV})$}

Size and zeta potential of the loaded niosomes were presented in Fig. (1). The $2.0 \% \mathrm{NV}$ gave the average size of $254 \pm 20.32 \mathrm{~nm}$, PDI of 0.322 and zeta potential of $50.07 \pm 3.11 \mathrm{mV}$ which was outside the range of $\pm 30 \mathrm{mV}$ range demonstrating the physical stability of the dispersion [18]. After stored at 4 and $25^{\circ} \mathrm{C}$ for 3 months, the loaded niosomes showed the range of size of about $230-280 \mathrm{~nm}$, while the niosomes at $45^{\circ} \mathrm{C}$ showed more fluctuate size of about 250-300 $\mathrm{nm}$. This might be due to the heat from the storage temperature changed the gel structure of niosomal membrane from the closely packed gel to the loosely packed liquid crystalline structure resulting in the variation of 
(A)

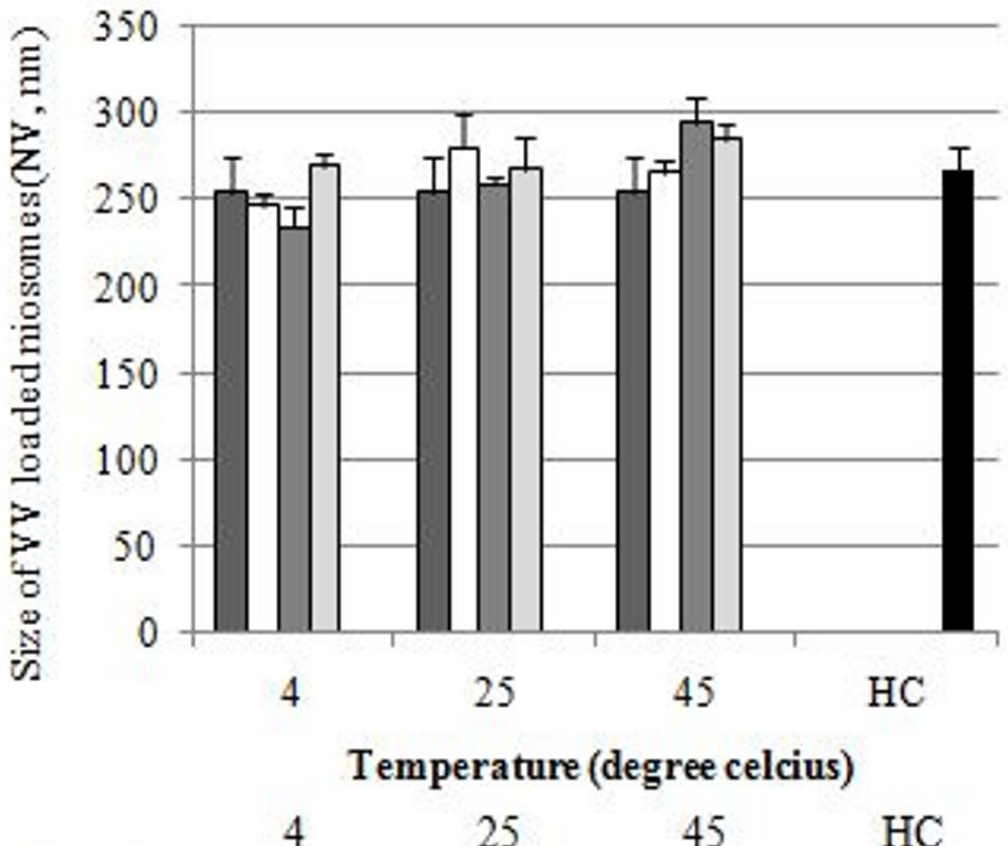

a month

$\square 1$ month

$\square 2$ months

प3 months

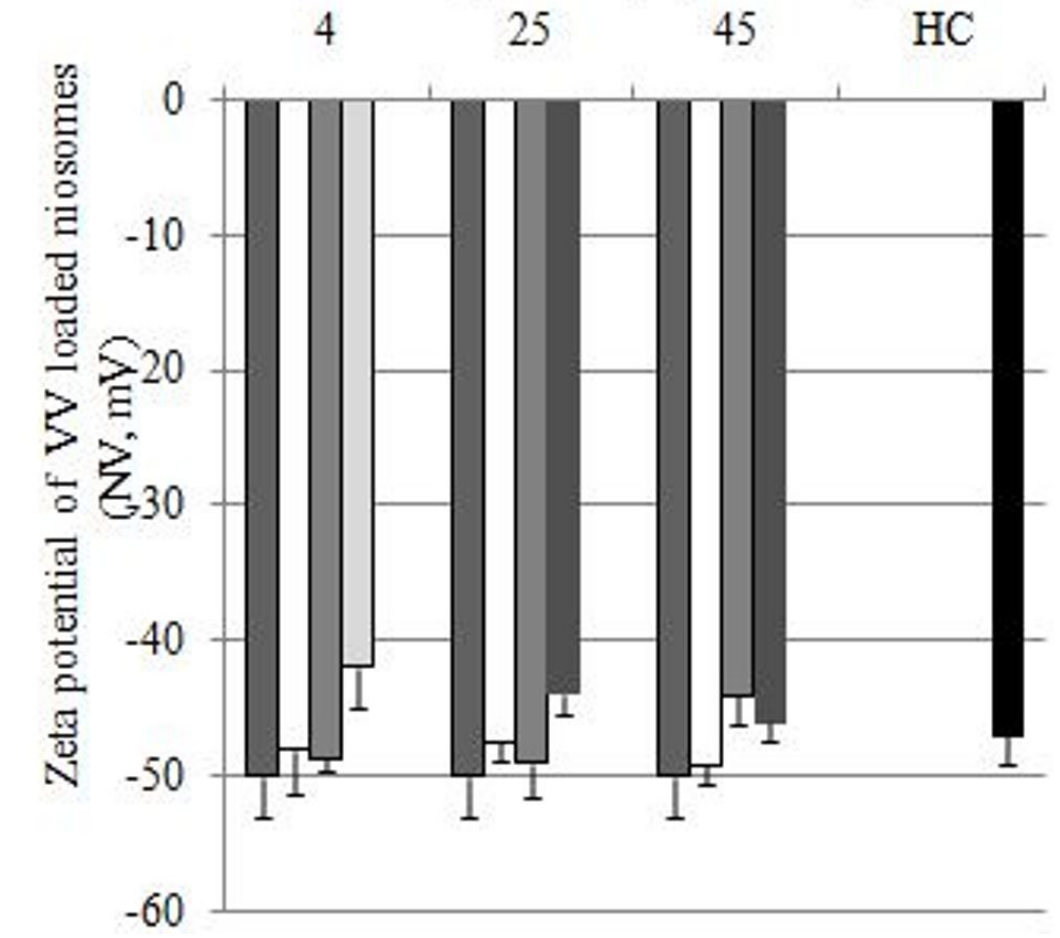

- heat-cool cycle

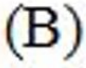

Fig. (1). The mean size (A) and zeta potential (B) value of niosomes loaded with VV extract (NV) at various storage temperature (4 $\pm 2,25 \pm 2$ and $45 \pm 2^{\circ} \mathrm{C}$ ) and heat cool cycle.

niosomal size [19]. However, the niosomal size after stored at three different temperatures for 3 months were in the acceptable range $(230-300 \mathrm{~nm})$. Moreover, the VV loaded in niosomes gave acceptable negatively zeta potential values of about -44 to $-50 \mathrm{mV}$ in all temperature conditions throughout 3 months.

\section{Chemical Characteristic of Niosomes Loaded with VV Extract (NV)}

The relative amount of total phenolic content in various formulations containing VV extract at 3 different storage temperatures $\left(4 \pm 2,25 \pm 2\right.$ and $\left.45 \pm 2^{\circ} \mathrm{C}\right)$ for 3 months were shown in Fig. (2). Solution of VV (SV) showed the lowest amount of total phenolic compound throughout 3 months. After 3 months of storage, SV at $45 \pm 2{ }^{\circ} \mathrm{C}$ gave the total phenolic content at $64.21 \pm 4.56 \%$ of initial. This may be due to the influence from the high temperature and exposure time that affect the total phenolic content of VV extract in SV. When VV extract was loaded in niosomes to enhance the stability of VV, niosomes loaded with VV (NV) gave more remaining amount total phenolic compound of $1.26,1.32$ and 1.33 times than in $\mathrm{SV}$ at $4 \pm 2,25 \pm 2$ and $45 \pm 2^{\circ} \mathrm{C}$ in the $3^{\text {rd }}$ month, respectively. Both $\mathrm{NV}$ and gel containing the 


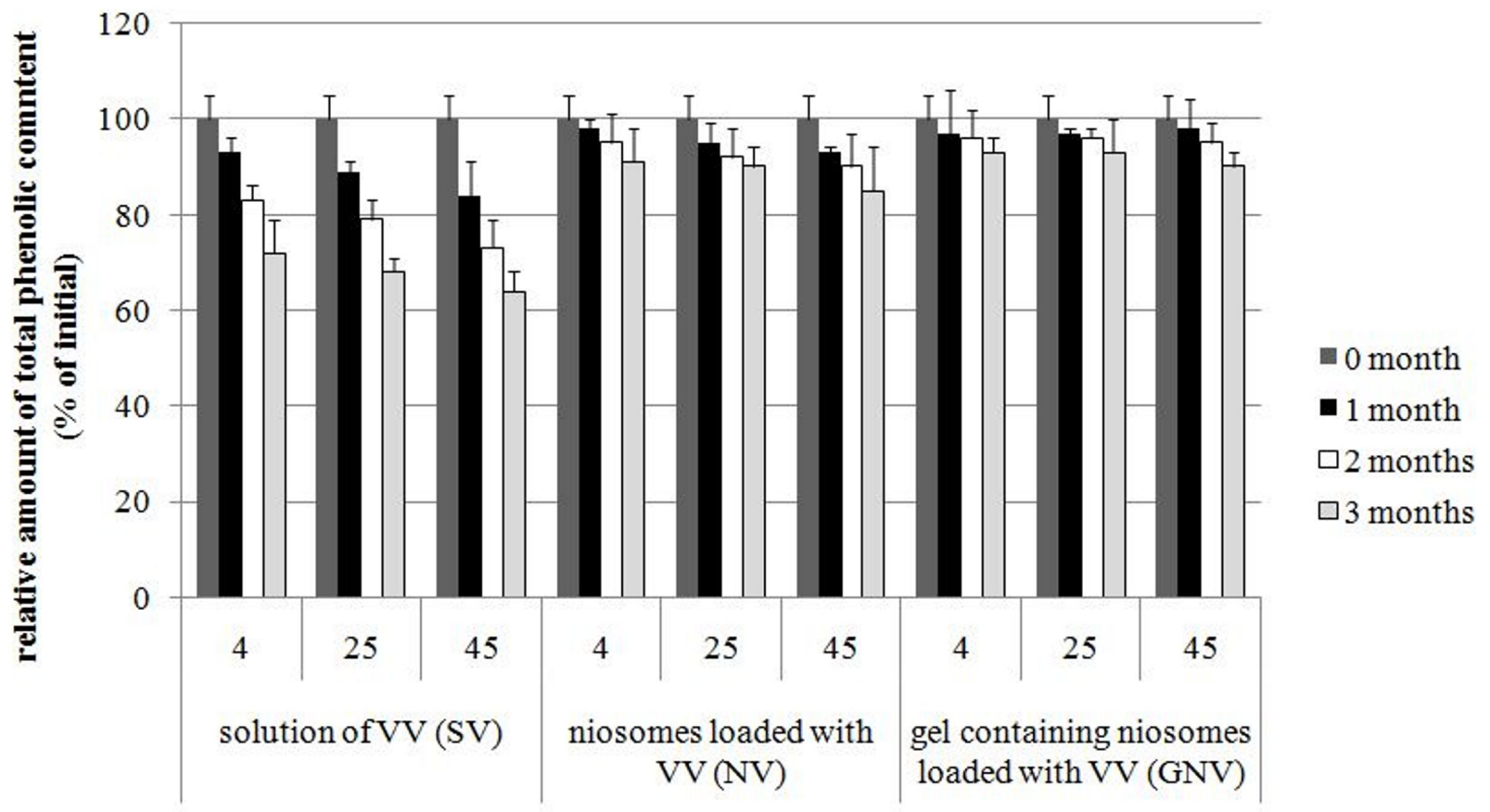

Temperature (degree celcius)

Fig. (2). Chemical stability of total phenolic content in various formulations containing VV extract at 3 different storage temperatures (4 \pm 2 , $25 \pm 2$ and $\left.45 \pm 2^{\circ} \mathrm{C}\right)$.

niosomes loaded with VV (GNV) gave more than $80 \%$ total phenolic content remaining through the stability period of 3 months at 3 different temperatures indicating the acceptable stability of total phenolic content after loading in niosomes. However, GNV showed higher content of total phenolic compound than those in NV of about 1.02, 1.03 and 1.05 times at $4 \pm 2,25 \pm 2$ and $45 \pm 2^{\circ} \mathrm{C}$ in the $3^{\text {rd }}$ month, respectively, indicating the double environment protections from niosomes and also the gel structure that protects $\mathrm{VV}$ extract from the environment [20].

\section{Transdermal Absorption of Various Formulations Containing VV Extract}

The cumulative amounts of total phenolic compound in various systems in whole skin and the receiving compartment solution through transdermal absorption across the excised rat skin were shown in Fig. (3). The cumulative amounts of total phenolic compound in all systems gradually increased in both whole skin and receiving compartment solution throughout the period of 6 hours except the SV, which was stable after the $1^{\text {st }}$ and $4^{\text {th }}$ hour of the experiment. This may be due to the effect of the saturation of $\mathrm{VV}$ extract in the whole skin and then it was released to the receiving compartment solution, resulting in the gradually increasing of the cumulative amounts of total phenolic compound in the receiving compartment solution of $\mathrm{SV}$ system. The same mechanism, the VV extract saturation in whole skin and then release to the receiving compartment, was occurred in $\mathrm{NV}$ and GNV system. But the VV extract saturation did not cease the cumulative amounts of total phenolic compound in the whole skin, the cumulative amounts of total phenolic compound of NV and GNV were gradually decreased, which was agreed with the previous study [21]. This might be the advantage of niosomes in the transdermal delivery [22] that can load the substance inside them and penetrate through the skin via like dissolve like theory. Since the niosomes composition, cholesterol was found in SC [23]. The relative amount of total phenolic content from various systems containing VV extract (SV, NV and GNV) which delivered through vertical Franz diffusion cells comparing to the initial loading amount were shown in Fig. (4). The increasing rate of the relative amount of initial loading from NV and GNV were higher than SV. This may be from the slow release of niosomes that gradually released $\mathrm{VV}$ extract from the systems to the environment. But SV system showed the relative amount of total phenolic compound in the $1^{\text {st }}$ hour in whole skin and the receiving compartment solution at about 4 and $1.75 \%$ which were higher than those from NV system of about 1.74 and 2.75 times, respectively. This might be from the retard effect of niosomes system that cannot promptly release the substance to the environment as much as SV system. However, NV and GNV gave the retard effect at the $1^{\text {st }}$ hour, but they could deliver higher total phenolic content than SV system at the $6^{\text {th }}$ hour of about 1.45 and 1.38 times in the whole skin and 1.48 and 1.68 times in the receiving compartment solution, respectively. Moreover, NV system gave the highest amount of total phenolic compound in the whole skin at the $6^{\text {th }}$ hour at $10.25 \%$ of initial, while GNV gave the highest amount of total phenolic compound in 
(A)

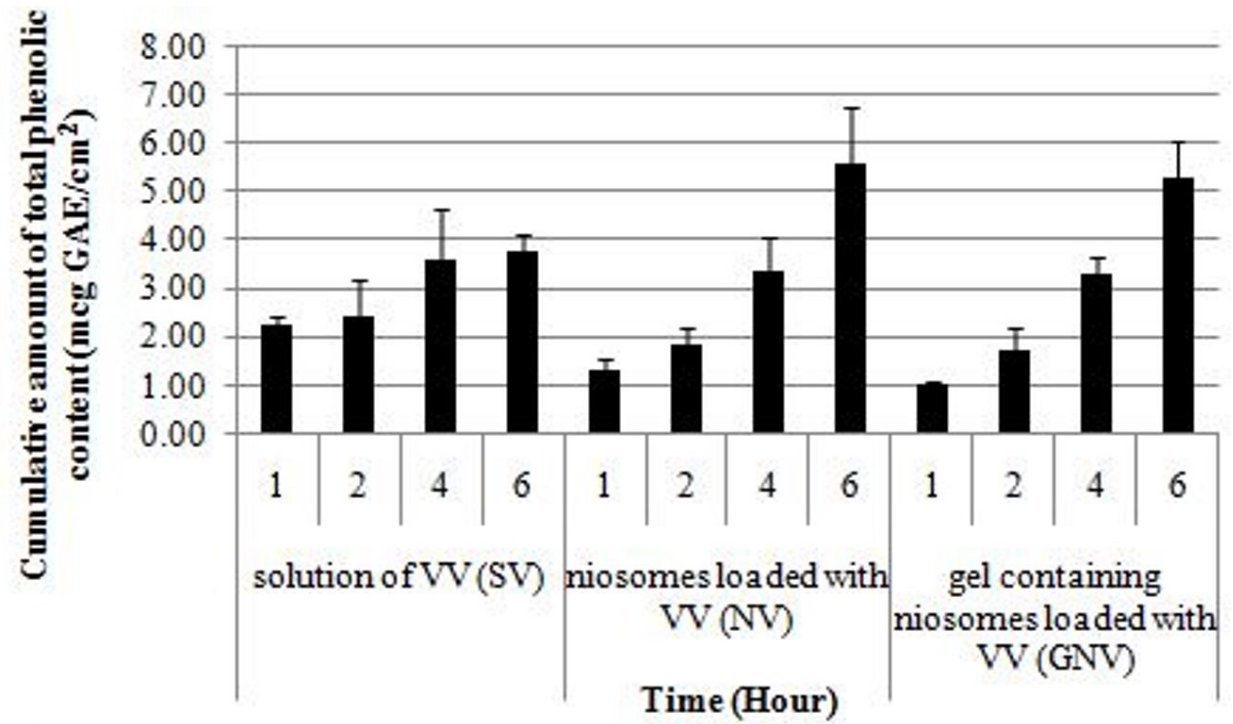

(B)

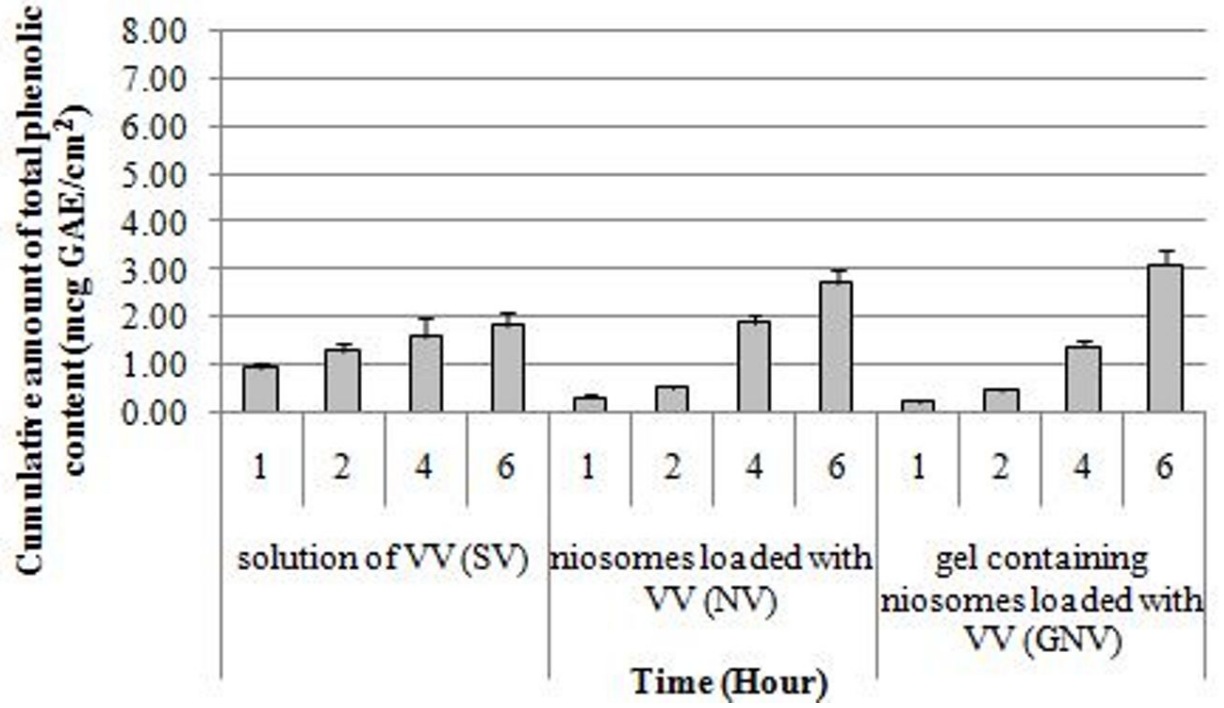

Fig. (3). The cumulative amounts $\left(\mathrm{mcg} \mathrm{GAE} / \mathrm{cm}^{2}\right)$ of total phenolic content from various systems containing VV extract in whole skin (A) and receiving compartment solution (B) through transdermal absorption across excised rat skin.

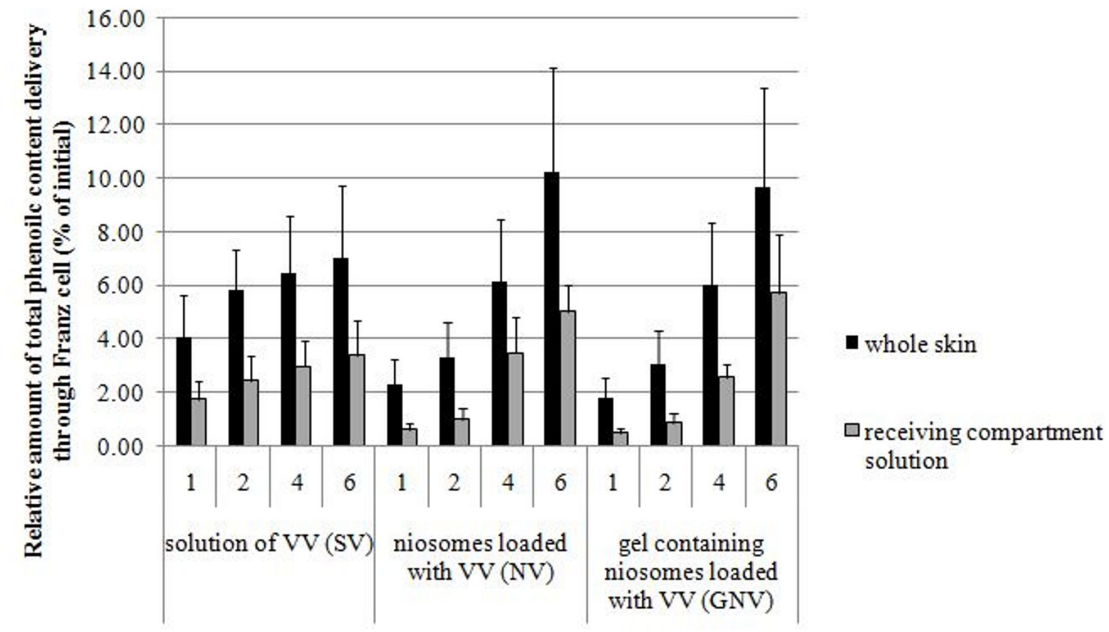

Time (Hour)

Fig. (4). Relative amount of total phenolic content from various systems containing VV extract (SV, NV and GNV) which delivered through vertical Franz diffusion cells comparing to the initial loading amount. 
Table 1. The cumulative amounts $\left(\mathrm{mcg} \mathrm{GAE} / \mathrm{cm}^{2}\right)$ and fluxes $\left(\mathrm{mcg} / \mathrm{cm}^{2} / \mathrm{h}\right)$ of total phenolic content from various systems containing VV extract in whole skin and receiving compartment solution through transdermal absorption across excised rat skin at $6 \mathrm{~h}$ by vertical Franz diffusion cells.

\begin{tabular}{|c|c|c|c|c|c|c|c|c|c|c|c|c|}
\hline \multirow{3}{*}{$\begin{array}{c}\text { Samples } \\
\text { solution of VV (SV) }\end{array}$} & \multicolumn{6}{|c|}{ Cumulative Amounts (mcg GAE $/ \mathrm{cm}^{2}$ ) } & \multicolumn{6}{|c|}{ Fluxes $\left(\mathrm{mcg}\right.$ GAE $\left./ \mathrm{cm}^{2} / \mathrm{h}\right)$} \\
\hline & \multicolumn{3}{|c|}{ Whole Skin } & \multicolumn{3}{|c|}{$\begin{array}{c}\text { Receiving Compartment } \\
\text { Solution }\end{array}$} & \multicolumn{3}{|c|}{ Whole Skin } & \multicolumn{3}{|c|}{$\begin{array}{c}\text { Receiving Compartment } \\
\text { Solution }\end{array}$} \\
\hline & 3.714 & \pm & 0.412 & 1.846 & \pm & 0.265 & 0.619 & \pm & 0.069 & 0.308 & \pm & 0.044 \\
\hline niosomes loaded with VV (NV) & 5.565 & \pm & $1.141^{*}$ & 2.735 & \pm & 0.274 & 0.928 & \pm & $0.190 *$ & 0.456 & \pm & 0.046 \\
\hline $\begin{array}{c}\text { gel containing niosomes loaded } \\
\text { with VV (GNV) }\end{array}$ & 5.260 & \pm & 0.783 & 3.108 & \pm & $0.311^{*}$ & 0.877 & \pm & 0.131 & 0.518 & \pm & $0.052 *$ \\
\hline
\end{tabular}

Notes: Each value represented mean \pm S.D. of three experiments.

$*$ : significant difference $(p<0.05)$ in comparing to the SV system.

the receiving compartment receiving compartment solution at the $6^{\text {th }}$ hour at $5.72 \%$ of initial (Fig. 4). The fluxes at the $6^{\text {th }}$ hour $\left(\mathrm{mcg} / \mathrm{cm}^{2} / \mathrm{h}\right)$ of various systems containing $\mathrm{VV}$ extract (SV, NV and GNV) that permeated the rat skin were calculated using the linear part of the correlation between the cumulative amounts of total phenolic compound by unit area and time were shown in Table 1 . The fluxes of the whole skin at the $6^{\text {th }}$ hour of NV system at $0.928 \pm 0.190 \mathrm{mcg} / \mathrm{cm}^{2} / \mathrm{h}$ was significantly different from that of $\mathrm{SV}$ at $0.619 \pm 0.069$ $\mathrm{mcg} / \mathrm{cm}^{2} / \mathrm{h}(p<0.05)$. This might conclude that niosomes are suitable for the penetration into the skin and remain in the skin which was not suitable for the delivery substance to the blood vessel, while gel system of GNV can deliver more substance to the receiving compartment solution with the significantly different flux of $0.518 \pm 0.052 \mathrm{mcg} / \mathrm{cm}^{2} / \mathrm{h}$ $(p<0.05)$, that represented for the blood vessels that may from the occlusion effect of gel [24]. This study has suggested that the gel containing the niosomes loaded with VV (GNV) appeared to be the most suitable system to be used for topical anti-aging application with the highest transdermal penetration of total phenolic compound into the receiving compartment than niosomes system, the proper viscosity for convenient topical use and superior occlusion effect of the gel that will be beneficial for the saturation in the skin.

\section{CONCLUSION}

The sonicated aqueous extracts of $V$. volvacea were loaded in neutral niosomes $(20 \mathrm{mM}$ of Tween 61 mixed with cholesterol at 1:1 molar ratio) to enhance the stability of loaded substance. The $2.0 \% \mathrm{w} / \mathrm{v}$ of niosomes loaded with VV extract (NV) gave the average size of $254 \pm 20.32 \mathrm{~nm}$ and zeta potential of $-50.07 \pm 3.11 \mathrm{mV}$ demonstrating the good physical stability of the dispersion. Gel containing the niosomes loaded with VV (GNV) was prepared. . Both NV and GNV gave more than $80 \%$ total phenolic content remaining through the stability period of 3 months at 3 different temperatures indicating the acceptable stability of total phenolic content after loading in niosomes. NV system gave the significantly highest amount of total phenolic compound in the whole skin at the $6^{\text {th }}$ hour at the relative amount of $10.25 \%$ of initial and the flux of $0.928 \pm 0.190$ $\mathrm{mcg} / \mathrm{cm}^{2} / \mathrm{h}$, while GNV gave the highest amount of total phenolic compound in the receiving compartment receiving compartment solution at the $6^{\text {th }}$ hour at $5.72 \%$ of initial and the flux of $0.619 \pm 0.069 \mathrm{mcg} / \mathrm{cm}^{2} / \mathrm{h}$ showing that niosomes are suitable for the penetration into and remain in the skin, while gel system can deliver more substance to the receiving compartment solution. This study has suggested that the gel containing the niosomes loaded with VV appeared to be the most suitable system to be used for topical anti-aging application with the highest transdermal penetration of total phenolic compound into the receiving compartment, the proper viscosity for convenient topical use and superior occlusion effect that will be beneficial for the saturation in the skin.

\section{CONFLICT OF INTEREST}

The authors confirm that this article content has no conflict of interest.

\section{ACKNOWLEDGEMENTS}

This work was supported by the 2013 grant from the Faculty of Pharmacy, Chiang Mai University, Chiang Mai, Thailand and the grant from the Chiang Mai University, Chiang Mai, Thailand.

\section{REFERENCES}

[1] Ruksiriwanich W, Sirithunyalug J, Boonpisuttinant K, Jantrawut P. Potent in vitro collagen biosynthesis stimulating and antioxidant activities of edible mushroom Volvariella volvacea aqueous extract. Int J Pharm Pharm Sc 2014; 6: 406-12.

[2] Barros L, Ferreira M, Queiros B, Ferreira I, Baptista P. Total phenols, ascorbic acid, $\beta$-carotene and lycopene in Portuguese wild edible mushrooms and their antioxidant activities. Food Chem 2007; 103: 413-9.

[3] Turkoglu A, Duru M, Mercan N, Kivrak I, Gezer K. Antioxidant and antimicrobial activities of Laetiporous sulphureus (Bull.) Murrill. Food Chem 2007; 101: 267-73.

[4] Agarwal R, Katare OP, Vyas SP. Preparation and in vitro evaluation of liposomal/niosomal delivery systems for antipsoriatic drug dithranol. Int J Pharm 2001; 228: 43-52.

[5] Knepp VM, Jr FCS, Guy RH. Controlled drug release from a novel liposomal delivery system. II. Transdermal delivery characteristics. J Controlled Release 1990; 12: 25-30.

[6] Biswal S, Murthy P, Sahu J, Sahoo P, Amir F. Vesicles of nonionic surfactants (niosomes) and drug delivery potential. Int $\mathbf{J}$ Pharm Sci Nanotech 2008; 1: 1-8.

[7] Marianecci C, Di Marzio L, Rinaldi F, et al. Niosomes from 80s to present: the state of the art. Adv Colloid Interface Sci 2014; 205: 187-206.

[8] Moghassemi S, Hadjizadeh A. Nano-niosomes as nanoscale drug delivery systems: an illustrated review. J Control Release 2014; 185: 22-36. 
[9] Yuan JS, Ansari M, Samaan M, Acosta EJ. Linker-based lecithin microemulsions for transdermal delivery of lidocaine. Int J Pharm 2008; 349: 130-43.

[10] Choi J, Choi M-K, Chong S, Chung S-J, Shim C-K, Kim D-D. Effect of fatty acids on the transdermal delivery of donepezil: in vitro and in vivo evaluation. Int J Pharm2012; 422: 83-90.

[11] Hathout RM, Woodman TJ, Mansour S, Mortada ND, Geneidi AS, Guy RH. Microemulsion formulations for the transdermal delivery of testosterone. Eur J Pharm Sci 2010; 40: 188-96.

[12] Chaiyana W, Saeio K, Hennink WE, Okonogi S. Characterization of potent anticholinesterase plant oil based microemulsion. Int $\mathbf{J}$ Pharm 2010; 401: 32-40.

[13] El Maghraby GM. Self-microemulsifying and microemulsion systems for transdermal delivery of indomethacin: effect of phase transition. Colloids Surf B 2010; 75: 595-600.

[14] Lvovich VF, Matthews E, Riga AT, Kaza L. AC electrokinetic platform for iontophoretic transdermal drug delivery. J Control Release 2010; 145: 134-40.

[15] Gadhiya P, Shukla S, Modi D, Bharadia P. Niosomes in Targeted Drug Delivery - A Review. IJPRS 2012; 1: 59-72.

[16] Zhou K, Su L, Yu L. Phytochemicals and antioxidant properties in wheat bran. J Agric Food Chem 2004; 52: 6108-14.
[17] Ruksiriwanich W, Sringarm K, Jantrawut P. Stability enhancement of Celastrus paniculatus seed oil by loading in niosomes Asian J Pharm Clin Res 2014; 7: 186-91.

[18] Gibson N, Shenderova O, Luo TJM, et al. Colloidal stability of modified nanodiamond particles. DRM 2009; 18: 620-6.

[19] Mokhtar M, Sammour OA, Hammad MA, Megrab NA. Effect of some formulation parameters on flurbiprofen encapsulation and release rates of niosomes prepared from proniosomes. Int $\mathrm{J}$ Pharm 2008; 361: 104-11.

[20] Tavano L, Muzzalupo R, Picci N, de Cindio B. Co-encapsulation of antioxidants into niosomal carriers: gastrointestinal release studies for nutraceutical applications. Colloids Surf B 2014; 114: 82-88.

[21] Balakrishnan P, Shanmugam S, Lee WS, et al. Formulation and in vitro assessment of minoxidil niosomes for enhanced skin delivery. Int J Pharm 2009; 377: 1-8.

[22] Muzzalupo R, Tavano L, Cassano R, Trombino S, Ferrarelli T, Picci N. A new approach for the evaluation of niosomes as effective transdermal drug delivery systems. Eur J Pharm Biopharm 2011; 79: 28-35.

[23] Menon GK. New insights into skin structure: scratching the surface. Adv Drug Deliv Rev 2002; 54 (Suppl 1): S3-17.

[24] Suetake T, Sasai S, Zhen Y-X, Tagami H. Effects of silicone gel sheet on the stratum corneum hydration. Brit J Plast Surg 2000; 53: 503-7.

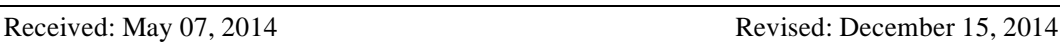

Accepted: December 22, 2014

(C) Shahirah et al.; Licensee Bentham Open.

This is an open access article licensed under the terms of the Creative Commons Attribution Non-Commercial License (http://creativecommons.org/licenses/by$\mathrm{nc} / 3.0 /$ ), which permits unrestricted, non-commercial use, distribution and reproduction in any medium, provided the work is properly cited 